\title{
Excited-State Proton-Transfer-Induced Trapping Enhances the Fluorescence Emission of a Locked GFP Chromophore
}

\author{
Xiang-Yang Liu, ${ }^{\dagger}$ Xue-Ping Chang, ${ }^{\dagger}$ Shu-Hua Xia, ${ }^{\dagger}$ Ganglong Cui, ${ }^{*}{ }^{\dagger}$ and Walter Thiel ${ }^{*}$, \\ ${ }^{\dagger}$ Key Laboratory of Theoretical and Computational Photochemistry, Ministry of Education, College of Chemistry, Beijing Normal \\ University, Beijing 100875, China and \\ ${ }^{\ddagger}$ Max-Planck-Institut für Kohlenforschung, Kaiser-Wilhelm-Platz 1, 45470 Mülheim an der Ruhr, Germany
}

Supporting Information

\begin{abstract}
The chemical locking of the central single bond in core chromophores of green fluorescent proteins (GFPs) influences their excited-state behavior in a distinct manner. Experimentally, it significantly enhances the fluorescence quantum yield of GFP chromophores with an ortho-hydroxyl group, while it has almost no effect on the photophysics of GFP chromophores with a para-hydroxyl group. To unravel the underlying physical reasons for this different behavior, we report static electronic structure calculations and nonadiabatic dynamics simulations on excited-state intramolecular proton transfer, cis-trans isomerization, and excited-state deactivation in a locked ortho-substituted GFP model chromophore (o-LHBI). On the basis of our previous and present results, we find that the $S_{1}$ keto species is responsible for the fluorescence emission of the unlocked o$\mathrm{HBI}$ and the locked o-LHBI species. Chemical locking does not change the parts of the $S_{1}$ and $S_{0}$ potential energy surfaces relevant to enol-keto tautomerization; hence, in both chromophores, there is an ultrafast excited-state intramolecular proton transfer that takes only $35 \mathrm{fs}$ on average. However, the locking effectively hinders the $S_{1}$ keto species from approaching the keto $S_{1} / S_{0}$ conical intersections so that most of trajectories are trapped in the $S_{1}$ keto region for the entire 2 ps simulation time. Therefore, the fluorescence quantum yield of o-LHBI is enhanced compared with that of unlocked o-HBI, in which the $S_{1}$ excited-state decay is efficient and ultrafast. In the case of the para-substituted GFP model chromophores $\mathrm{p}$-HBI and p-LHBI, chemical locking hardly affects their efficient excited-state deactivation via cis-trans isomerization; thus, the fluorescence quantum yields in these chromophores remain very low. The insights gained from the present work may help to guide the design of new GFP chromophores with improved fluorescence emission and brightness.
\end{abstract}

\section{INTRODUCTION}

Green fluorescent proteins (GFPs) have received a great deal of attention due to their ubiquitous application in molecular biology. ${ }^{1-7}$ Their high fluorescence quantum yields make them powerful tools in bioimaging technology. Due to their importance in real applications, a broad range of GFP variants has been developed in the past decades, but their brightness and stability are still not optimal. ${ }^{8,9}$ To improve their overall performance, numerous experimental and theoretical studies have in the past decades been employed to investigate the working mechanism of both the natural GFPs and chemically synthesized GFP core chromophores. ${ }^{10-29}$

Natural GFP core chromophores with a para-hydroxyl group such as 4-(4-hydroxybenzylidene)-1,2-dimethyl-1H-imidazol$5(4 \mathrm{H})$-one (p-HBDI) are not emissive in solution, neither in their neutral nor in their anionic form. This nonemissive character can mostly be attributed to an ultrafast nonadiabatic decay induced by rotation around the exocyclic $\mathrm{C}=\mathrm{C}$ double bond that leads to cis-trans isomerization. This viewpoint has been confirmed by many experimental and theoretical studies including nonadiabatic dynamical simulations. ${ }^{30-36}$ Recently,
GFP core chromophores with an ortho-hydroxyl group have come into the focus of experimental research, for example 4-(2hydroxybenzylidene)-1,2-dimethyl-1H-imidazol-5(4H)-one (oHBDI). ${ }^{37-40}$ This ortho-substitution allows the formation of an intramolecular hydrogen bond as part of a seven-membered ring, which causes a qualitatively different excited-state behavior. Hsieh et al. studied the photophysics and photochemistry of o-HBDI and its analogues using various ultrafast spectroscopic techniques. ${ }^{39}$ They observed an ultrafast excitedstate intramolecular proton transfer from the hydroxyl to the imidazole group, in less than $25 \mathrm{fs}$, and a very low cis-trans photoisomerization quantum yield of ca. 5\%. This is totally different from the excited-state behavior of the natural GFP core chromophore, in which there is no excited-state intramolecular proton transfer and the cis-trans quantum yield approaches $50 \%$. Furthermore, the quantum yield of the fluorescence emission in o-HBDI was found to be rather low, ca. $3.3 \times 10^{-3}$ in toluene. ${ }^{39}$ This intriguing excited-state

Received: September 17, 2015

Published: January 8, 2016 
dynamics was explained with the use of high-level static electronic structure calculations and nonadiabatic dynamics simulations on 4-(2-hydroxybenzylidene)-1H-imidazol-5 $(4 \mathrm{H})$ one (o-HBI). ${ }^{41}$ According to this theoretical study, the $S_{1}$ state of o-HBI is of charge-transfer character, which facilitates the excited-state proton transfer, and the low cis-trans quantum yield is due to the fact that the $S_{1} / S_{0}$ conical intersection responsible for the $S_{1}$ excited-state deactivation is close to the cis region; this scenario was verified by nonadiabatic dynamics simulations. ${ }^{41}$ In view of these experimental and computational results, one can conclude that o-HBDI and its variants are not able to serve as GFP core chromophores due to the ultralow quantum yield of fluorescence emission.

However, simple chemical tailoring can remarkably enhance the fluorescence emission of o-HBDI. Motivated by the concept that structural rigidity may help to increase the quantum yield of fluorescence emission, $\mathrm{Hsu}$ et al. recently synthesized the structurally locked para- and ortho-hydroxyl GFP core chromophores, p-LHBDI and o-LHBDI (Figure 1),<smiles>CC1=[N+]([O])C(=Cc2ccccc2O)C(=O)N1C1=N/C(=C\c2ccc(O)cc2)C(=O)N1C</smiles>

o-HBDI $\left(3.3^{*} 10^{-3}\right)$

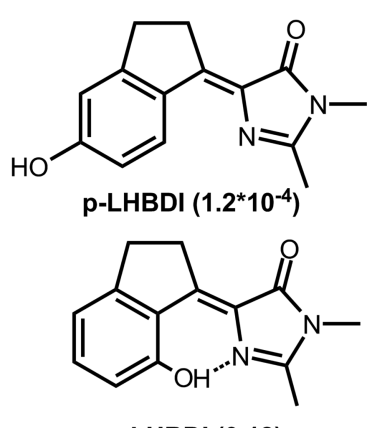

o-LHBDI (0.18)
Figure 1. GFP core chromophores with para- and ortho-hydroxyl groups studied experimentally and measured quantum yields of fluorescence emission (in parentheses).

in which rotation around the $\mathrm{C} 3-\mathrm{C} 4$ bond is not possible. ${ }^{40}$ An especially interesting experimental finding is that the locking causes a distinctly different excited-state behavior in p-LHBDI and o-LHBDI. It significantly enhances the quantum yield of fluorescence emission of the ortho-hydroxyl GFP chromophores, e.g. $3.3 \times 10^{-3}$ for o-HBDI versus 0.18 for o-LHBDI. By contrast, it makes no difference to the quantum yield of fluorescence emission of the para-hydroxyl GFP chromophores, ca. $10^{-4}$ of $\mathrm{p}$-HBDI versus $1.2 \times 10^{-4}$ of p-LHBDI. ${ }^{40}$ Undoubtedly, a thorough understanding of the effects of chemical tailoring on the excited-state dynamics of GFP core chromophores is important not only for enriching our knowledge on GFP but also for guiding the design of new GFP chromophores with improved performance and brightness.

In this article, we employ a series of high-level electronic structure methods (DFT, CASSCF, and MS-CASPT2) to map the $S_{1}$ and $S_{0}$ potential energy profiles of a truncated o-LHBDI model (o-LHBI, without the two methyl groups) that are relevant to the intramolecular proton/hydrogen transfers, the excited-state deactivation paths, and the cis-trans isomerizations. Thereafter, we perform semiempirical OM2/MRCI nonadiabatic dynamics simulations of the $S_{1}$ photodynamics. On the basis of the previous and present results, we discuss the factors enhancing the quantum yield of fluorescence emission of o-LHBDI, and we rationalize why the chemical tailoring does not change the photophysics of p-LHBDI compared with unlocked p-HBDI.

\section{COMPUTATIONAL METHODS}

Ab Initio Calculations. Ground-state conformers of oLHBI were first optimized using density functional theory $(\mathrm{DFT})^{42}$ with the B3LYP exchange-correlation functional. ${ }^{4-46}$ Minima, conical intersections, minimum-energy potential energy profiles, and nudged elastic band (NEB) paths were optimized using the state-averaged complete active space selfconsistent field (CASSCF) method with equal state weights. In the CASSCF $(10,8)$ computations, the active space consisted of 10 electrons in 8 orbitals, which included eight $\pi$ electrons in seven $\pi$ and $\pi^{*}$ orbitals and two lone-pair electrons in one $n$ orbital (see Supporting Information, Figure S1). This active space provides a good balance between accuracy and efficiency in CASSCF excited-state geometry optimizations of mediumsize molecules. ${ }^{47,48}$

Since the CASSCF method does not adequately capture dynamic correlation, the multistate complete active space second-order perturbation approach (MS-CASPT2 $)^{49,50}$ was utilized to re-evaluate the energies of all optimized structures, minimum-energy potential energy profiles, and NEB paths. In the MS-CASPT2 computations, the Cholesky decomposition technique with unbiased auxiliary basis sets was used for accurate two-electron integral evaluation; ${ }^{51}$ the ionization potential-electron affinity (IPEA) shift was not applied, ${ }^{52}$ whereas the imaginary shift technique $(0.2 \mathrm{au})$ was employed to avoid intruder-state issues. ${ }^{53}$

Vertical excitation energies at Franck-Condon points were calculated using the MS-CASPT2 method and the timedependent DFT (TD-DFT) method at the TD-CAM-B3LYP level. $^{54,55}$ The 6-31G* basis set was employed throughout. ${ }^{56,57}$ DFT and TD-DFT computations as well as CASSCF optimizations of minima and conical intersections were carried out using GAUSSIAN09; ${ }^{42,54,58}$ SA-CASSCF calculations of minimum-energy potential energy profiles and MS-CASPT2 computations were performed using MOLCAS8.0; ${ }^{59,60}$ SACASSCF computations of NEB paths were done with MOLPRO2010. ${ }^{61}$

Semiempirical Calculations. All semiempirical calculations were performed using the OM2/MRCI method (OM2: orthogonalization model 2; MRCI multireference configuration interaction) as implemented in the MNDO99 code. $^{62-65}$ During geometry optimizations and dynamics simulations, all required energies, gradients, and nonadiabatic coupling elements were computed analytically. Minimum-energy conical intersections were optimized using the Lagrange-Newton approach. ${ }^{66,67}$

In the OM2/MRCI calculations, the restricted open-shell Hartree-Fock formalism was applied in the self-consistent field (SCF) treatment. The active space in the MRCI calculations included 14 electrons in 13 orbitals (see Supporting Information, Figure S2). In terms of the SCF configuration, it comprised the six highest doubly occupied orbitals, the two singly occupied orbitals, and the five lowest unoccupied orbitals. For the MRCI treatment, three configuration state functions were chosen as references, namely the SCF configuration and the two closed-shell configurations derived therefrom (i.e., all singlet configurations that can be generated from HOMO and LUMO of the closed-shell ground state). The MRCI wave function was built by allowing all single and double excitations from these three references. 
Nonadiabatic Dynamics Simulations. The excited-state nonadiabatic dynamics were studied by performing 2 ps OM2/ MRCI trajectory surface-hopping simulations. ${ }^{68-80}$ The initial atomic coordinates and velocities for the $S_{1}$ photodynamics simulations were randomly selected from a 5 ps NVT groundstate trajectory ( $T=300 \mathrm{~K}$, velocity-rescaling thermostat). A total of 275 snapshots were chosen from a larger randomly selected set on the basis of the computed $S_{0}-S_{1}$ transition probabilities. Starting from these snapshots, surface-hopping trajectories (NVE) were run for the $S_{1}$ photodynamics, with all relevant energies, gradients, and nonadiabatic coupling vectors being computed on-the-fly as needed, as described previously. ${ }^{70}$ For points with an $S_{0}-S_{1}$ energy gap of less than $10 \mathrm{kcal} / \mathrm{mol}$, the fewest-switches criterion was applied to decide whether to hop. The time step was chosen to be 0.1 fs for the nuclear motion and $0.0005 \mathrm{fs}$ for the electronic propagation. The unitary propagator evaluated at midpoint was used to propagate the electronic motion. The translational and rotational motions were removed in each step. A standard empirical decoherence correction $(0.1 \mathrm{au})$ was applied. ${ }^{81}$ The final evaluations were done for the 261 trajectories that finished successfully in the $S_{1}$ photodynamics runs and that satisfied our energy continuity criterion (no change greater than $30 \mathrm{kcal} / \mathrm{mol}$ between any two consecutive MD steps). Further technical details are given in previous publications.

\section{RESULTS AND DISCUSSION}

Minima in the $S_{0}$ and $S_{1}$ States. In the $S_{0}$ state, o-LHBI can be present as an enol tautomer (SO-ENOL) or as a keto tautomer (S0-KETO). The corresponding CASSCF-optimized equilibrium structures are shown in Figure 2. They are planar ( $C_{s}$ symmetry). The intramolecular $\mathrm{N} 1 \cdots \mathrm{H} 7-\mathrm{O} 6$ or $\mathrm{N} 1-\mathrm{H} 7 \cdots$ O6 hydrogen bonds and the locking by the two bridging carbon atoms both favor planarity.

Structurally, the central $\mathrm{C} 2-\mathrm{C} 3$ and $\mathrm{C} 3-\mathrm{C} 4$ bond lengths of S0-ENOL are computed to be 1.354 and $1.482 \AA$, respectively, indicating typical double- and single-bond character. When the $\mathrm{H} 7$ atom is transferred from the $\mathrm{O} 6$ to the $\mathrm{N} 1$ atom, the $\mathrm{C} 2-$
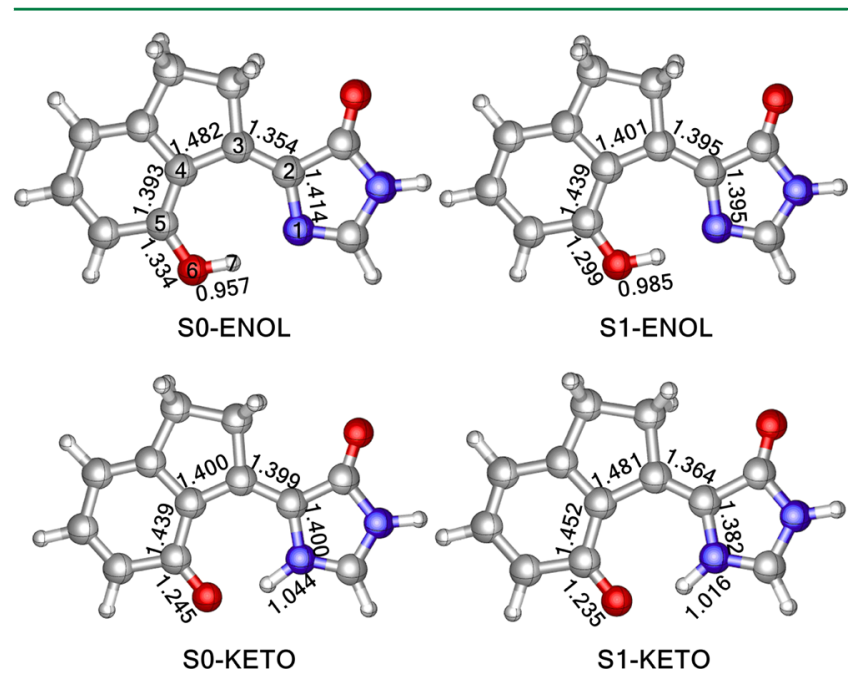

Figure 2. Optimized enol and keto minimum-energy structures in the $\mathrm{S}_{1}$ and $\mathrm{S}_{0}$ states. Also shown are selected bond lengths (in $\AA$ ) optimized at the $\operatorname{CASSCF}(10,8)$ level. The chosen atom numbering scheme is indicated in the case of S0-ENOL. See the Supporting Information for the OM2/MRCI geometrical parameters of these minima.
C3 [C3-C4] bond length increases [decreases] to 1.399 [1.400] $\AA$ in S0-KETO. The most significant structural change in the ground-state enol-keto tautomerization process is the marked shortening of the C5-O6 bond length, from $1.334 \AA$ in S0-ENOL to $1.245 \AA$ in SO-KETO. Energetically, S0-KETO is $12.0 \mathrm{kcal} / \mathrm{mol}$ higher than S0-ENOL at the MS-CASPT2 level.

In the $S_{1}$ state, the enol (S1-ENOL) and keto (S1-KETO) minima are structurally very different from their $S_{0}$ counterparts. Compared with $\mathrm{S} 0-\mathrm{ENOL}$, the $\mathrm{C} 2-\mathrm{C} 3$ bond is elongated by $0.041 \AA$ and the C3-C4 bond is shortened by $0.081 \AA$ in S1ENOL. The C5-O6 bond also becomes shorter, from $1.334 \AA$ in S0-ENOL to $1.299 \AA$ in S1-ENOL. Moreover, the O6-H7 ... $\mathrm{N} 1$ hydrogen-bonding is clearly enhanced in the $S_{1}$ state, as

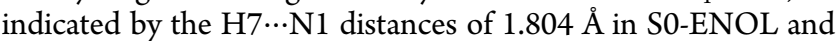
$1.665 \AA$ in S1-ENOL. By contrast, when going from the S0$\mathrm{KETO}$ to the S1-KETO minimum, the $\mathrm{C} 2-\mathrm{C} 3[\mathrm{C} 3-\mathrm{C} 4]$ bond length decreases [increases] by 0.035 [0.081] $\AA$, while the C5O6 bond length remains nearly unchanged, $1.245 \AA$ versus $1.235 \AA$. At the MS-CASPT2 level, S1-KETO is computed to be $4.7 \mathrm{kcal} / \mathrm{mol}$ lower in energy than S1-ENOL (see Table 1).

Table 1. Relative Energies ( $\mathrm{kcal} / \mathrm{mol}$ ) of Critical Structures at Different Computational Levels (OM2/MRCI, CASSCF, and MS-CASPT2//CASSCF) $)^{c}$

\begin{tabular}{lclc}
\multicolumn{1}{c}{ structure } & OM2/MRCI & CASSCF & MS-CASPT2//CASSCF $^{a}$ \\
S0-ENOL & 0.0 & 0.0 & 0.0 \\
S0-KETO & & 23.0 & 12.0 \\
S1-ENOL & & 92.3 & 70.0 \\
S1-KETO $^{2}$ & 63.2 & 86.9 & 65.3 \\
SOS1-ENOL-1 $^{b}$ & $49.5 / 49.5$ & $71.0 / 70.9$ & $63.5 / 52.1$ \\
SOS1-ENOL-2 $^{b}$ & $49.5 / 49.5$ & $71.1 / 70.8$ & $63.7 / 52.1$ \\
SOS1-KETO-1 $^{b}$ & $75.6 / 75.6$ & $91.6 / 88.6$ & $74.8 / 68.9$ \\
SOS1-KETO-2 $^{b}$ & $73.6 / 73.6$ & $95.3 / 92.2$ & $77.9 / 66.7$
\end{tabular}

${ }^{a}$ CASSCF optimized structures. ${ }^{b}$ Single-point energies for the two states $\left(S_{1} / S_{0}\right)$. ${ }^{c}$ See the Supporting Information for more detailed data.

We note that S0-KETO and S1-ENOL minima could not be located at the OM2/MRCI level because the corresponding tautomerizations are computed to be barrierless (see below). At the CASSCF level, S0-KETO and S1-ENOL are very shallow minima which disappear in single-point MS-CASPT2// CASSCF calculations (see below).

Local Spectroscopic Properties. Local spectroscopic properties are crucial for understanding the photodynamics of o-LHBI. Table 2 compiles the computed vertical excitation energies to the lowest excited singlet state at the enol FranckCondon point (SO-ENOL) and the vertical emission energies to the ground state at the $S_{1}$ keto minimum (S1-KETO). The calculated gas-phase excitation energies from OM2/MRCI and MS-CASPT2 are close to each other, 81.7 versus $82.1 \mathrm{kcal} / \mathrm{mol}$,

Table 2. Vertical Excitation Energies at the Enol FranckCondon Point (First Row) and Vertical Emission Energies at the Keto Franck-Condon Point (Second Row) of o-LHBI Computed at the MS-CASPT2//CASSCF and OM2/MRCI Levels (in $\mathrm{kcal} / \mathrm{mol}$ )

\begin{tabular}{lccc} 
& MS-CASPT2 & OM2/MRCI & $\exp ^{a}$ \\
\hline absorption & 82.1 & 81.7 & 73.5 \\
emission & 42.4 & 45.8 & 48.9
\end{tabular}

${ }^{a} \mathrm{O}$-LHBDI in toluene. 

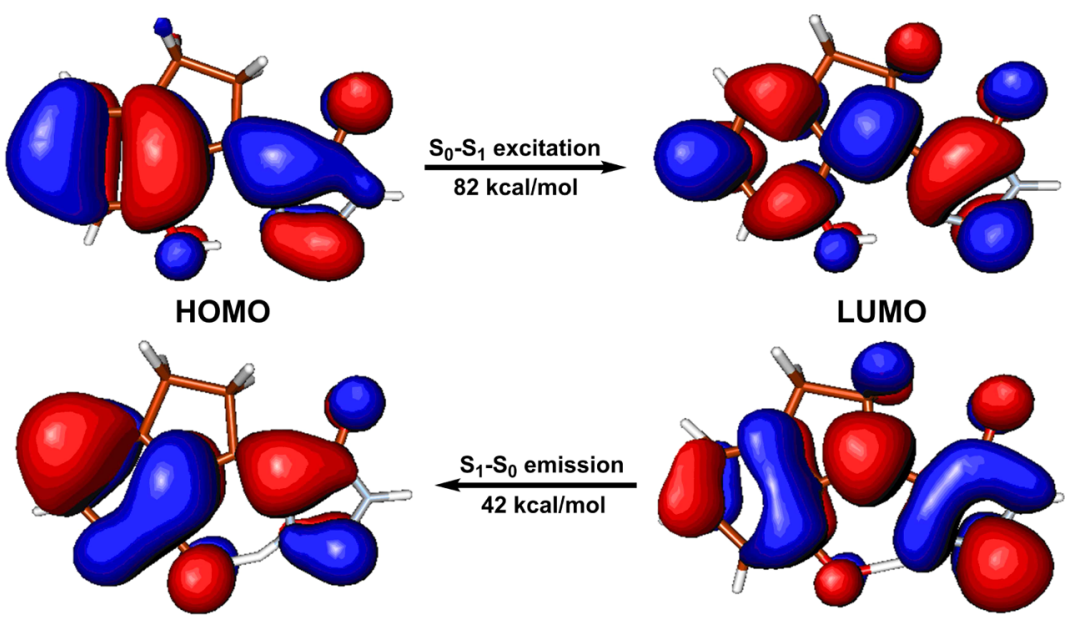

Figure 3. CASSCF computed molecular orbitals (MOs) involved in the electronic transition between the two lowest singlet states: (top) $S_{0} \rightarrow S_{1}$ excitation at the enol Franck-Condon point S0-ENOL; (bottom) $S_{1} \rightarrow S_{0}$ de-excitation at the keto Franck-Condon point S1-KETO. HOMO and LUMO denote the highest occupied and lowest unoccupied MO in the $S_{0}$ state, respectively.


Figure 4. Relaxed minimum-energy energy profiles for intramolecular proton transfer in the $\mathrm{S}_{1}$ state of o-LHBI computed with OM2/MRCI (left), CASSCF (middle), and MS-CASPT2//CASSCF (right). Also shown are the unrelaxed ground-state energy profiles obtained from corresponding $S_{0}$ single-point calculations.

and somewhat higher than the experimental value of $73.5 \mathrm{kcal} /$ mol measured in toluene solution (o-LHBDI). ${ }^{40}$ The computed gas-phase emission energies from OM2/MRCI and MS-CASPT2 (45.8 and $42.4 \mathrm{kcal} / \mathrm{mol}$ ) agree reasonably well with each other and with the experimental value $(48.9 \mathrm{kcal} /$ mol). The OM2/MRCI method thus provides realistic excitedstate energies, which is a prerequisite for using it to simulate the $\mathrm{S}_{1}$ photodynamics of o-LHBI.

Wave function analysis at the enol Franck-Condon point SO-ENOL shows that the $S_{1}$ electronic state is primarily composed of the HOMO $\rightarrow$ LUMO electronic configuration (see top panel of Figure 3). Evidently, the highest occupied molecular orbital (HOMO) and the lowest unoccupied molecular orbital (LUMO) have bonding and antibonding character between the $\mathrm{C} 2$ and $\mathrm{C} 3$ atoms, respectively. This character does not change when recomputing these orbitals at the $S_{1}$ keto minimum S1-KETO (see the bottom panel of Figure 3).

Enol-Keto Tautomerization. Relaxed minimum-energy reaction paths show that the $S_{1}$ enol-keto tautomerization in oLHBI is essentially barrierless (Figure 4). At the MSCASPT2//CASSCF level, the $S_{1}$ potential energy gradually decreases with decreasing $\mathrm{N} 1-\mathrm{H} 7$ distance (from S1-ENOL to S1-KETO). Qualitatively similar $S_{1}$ energy profiles are computed with CASSCF and OM2/MRCI (Figure 4). Singlepoint calculations along the relaxed $S_{1}$ paths yield unrelaxed $S_{0}$ energy profiles that rise when going from the enol to the keto species, confirming that the enol-keto tautomerization is unfavorable in the ground state. There is no $S_{1} / S_{0}$ crossing during tautomerization (Figure 4). Relaxed minimum-energy reaction paths for the $S_{0}$ enol-keto tautomerization are presented in the Supporting Information (Figures S4 and S5); again OM2/MRCI and CASSCF give similar energy profiles. Both at the OM2/MRCI and MS-CASPT2//CASSCF levels, the $S_{1}$ excited-state proton transfer and the $S_{0}$ groundstate hydrogen transfer are barrierless processes. At the CASSCF level, there is a small $S_{1}$ barrier of $1.9 \mathrm{kcal} / \mathrm{mol}$, which is expected to be of limited relevance for the excited-state dynamics because the $S_{1}$ energy at the Franck-Condon point is large enough to easily overcome this barrier.

Conical Intersections. Two kinds of $S_{1} / S_{0}$ minimumenergy conical intersections are optimized at both CASSCF and OM2/MRCI levels, which are labeled as S0S1-ENOL-1, S0S1ENOL-2, S0S1-KETO-1, and S0S1-KETO-2 (see Figure 5 for their CASSCF optimized structures). The two enol-type conical intersections have the same energy (see Table 1) and equivalent structures that differ qualitatively only in the sense of rotation around the $\mathrm{C} 2-\mathrm{C} 3$ bond between the imidazole ring and the five-membered ring (see Figure 5). In both cases, the imidazole ring is almost perpendicular to the fivemembered ring (C4C3C2N1 dihedral angle: $98.7^{\circ}$ for S0S1ENOL-1 and $-98.1^{\circ}$ for SOS1-ENOL-2), whereas the C5C4C3C2 moiety is essentially planar (C5C4C3C2 dihedral angle: $-1.4^{\circ}$ for S0S1-ENOL-1 and $1.1^{\circ}$ for SOS1-ENOL-2); 

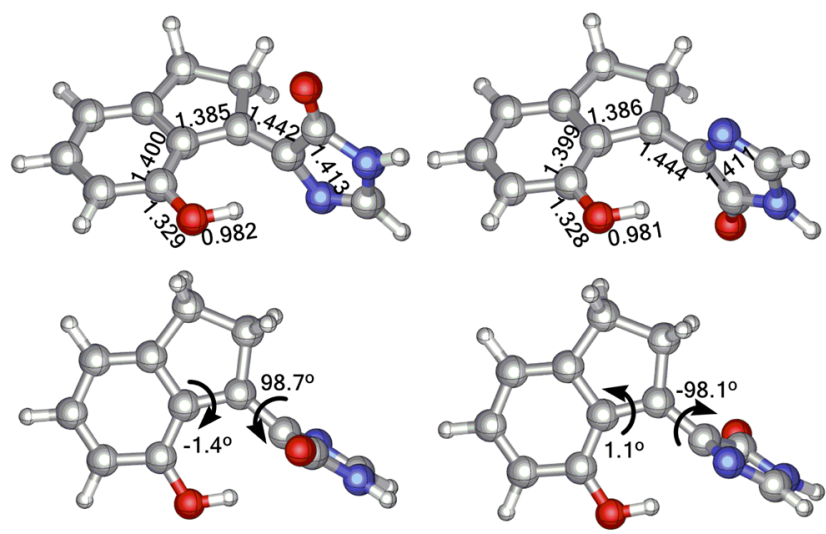

SOS1-ENOL-1
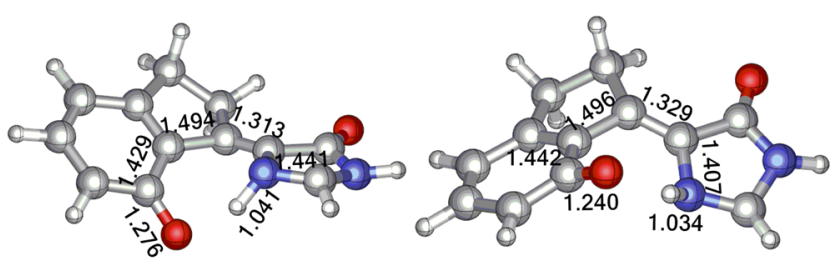

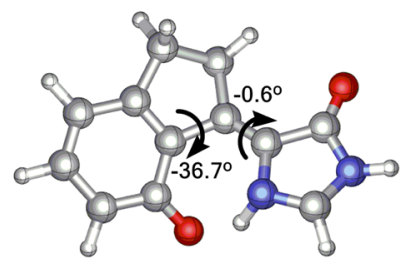

SOS1-KETO-1

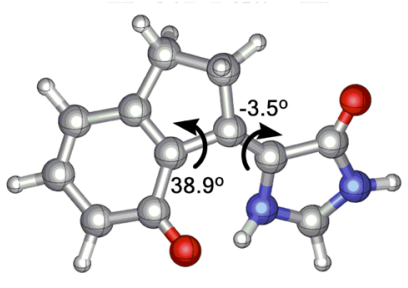

SOS1-KETO-2
Figure 5. Optimized structures of four $S_{1} / S_{0}$ minimum-energy conical intersections. Also shown are selected bond lengths (in $\AA$ ) and dihedral angles (in degree) optimized at the $\operatorname{CASSCF}(10,8)$ level. See the Supporting Information for the corresponding OM2/MRCI geometries.

the central $\mathrm{C} 2-\mathrm{C} 3$ bond is significantly longer than in the enol $\mathrm{S}_{1}$ minimum (e.g., $1.442 \AA$ of SOS1-ENOL-1 versus $1.395 \AA$ of S1-ENOL). At the MS-CASPT2 level, S0S1-ENOL-1 and SOS1-ENOL-2 are computed to be $12.2 \mathrm{kcal} / \mathrm{mol}$ (averaged between the $S_{1}$ and $S_{0}$ values) below the enol $S_{1}$ minimum ( $S 1$ ENOL) and $24.3 \mathrm{kcal} / \mathrm{mol}$ below the $S_{1}$ energy at the enol Franck-Condon point (S0-ENOL). The computed $S_{1}$ minimum-energy reaction paths with respect to the $\mathrm{C} 4 \mathrm{C} 3 \mathrm{C} 2 \mathrm{~N} 1$ dihedral angle show that the deactivation from
S1-ENOL to SOS1-ENOL-1 is energetically allowed (Figure 6). It is barrierless in the $S_{1}$ state at the OM2/MRCI level, while there are small barriers of ca. 5 and $3 \mathrm{kcal} / \mathrm{mol}$ at the CASSCF and MS-CASPT2//CASSCF levels; however, the deactivation remains efficient also in the latter two cases because the $S_{1}$ energies along the path are always below the $S_{1}$ energy at the Franck-Condon point ( $82 \mathrm{kcal} / \mathrm{mol}$ at the MS-CASPT2 level). Finally, we note that the $S_{1}$ and $S_{0}$ energy profiles computed with OM2/MRCI and CASSCF are similar in shape; in terms of the actual values, the OM2/MRCI energies are closer to the more accurate MS-CASPT2//CASSCF values (Figure 6).

The two keto-type conical intersections are also almost isoenergetic (see Table 1) and have structures that mainly differ in the sense of rotation around the $\mathrm{C} 3-\mathrm{C} 4$ bond in the fivemembered ring (see Figure 5). These structures are qualitatively different from their enol-type counterparts. The local environment of the $\mathrm{C} 2-\mathrm{C} 3$ bond remains almost planar in S0S1-KETO-1 and S0S1-KETO-2: the C4C3C2N1 dihedral angle is close to its value in the keto $S_{1}$ minimum S1-KETO (C4C3C2N1: $-0.6^{\circ}$ for S0S1-KETO-1 and $-3.5^{\circ}$ for S0S1$\mathrm{KETO}-2)$. The twist around the neighboring $\mathrm{C} 3-\mathrm{C} 4$ bond is restrained by the bridging carbon atoms; therefore, the C5C4C3C2 dihedral angle reaches only values of about $40^{\circ}$ in S0S1-KETO-1 and S0S1-KETO-2 (see bottom panel of Figure 5). The deactivation paths computed with CASSCF and $\mathrm{OM} 2 / \mathrm{MRCI}$ indicate that the lowest keto $S_{1} / S_{0}$ conical intersection SOS1-KETO-1 is accessible structurally (Figure 7) and also energetically (since all points lie below the $S_{1}$ energy at the enol Franck-Condon point). However, in both cases as well as at the MS-CASPT2//CASSCF level, the deactivation paths are uphill, with SOS1-KETO-1 being higher in energy than S1-KETO (MS-CASPT2//CASSCF: 71.9 (averaged between the $S_{1}$ and $S_{0}$ values) versus $65.3 \mathrm{kcal} /$ $\mathrm{mol}$; OM2/MRCI: 75.6 versus $63.2 \mathrm{kcal} / \mathrm{mol}$ ). Therefore, excited-state deactivation via the keto-type $S_{1} / S_{0}$ conical intersections is expected to be inefficient. This qualitatively rationalizes why the quantum yield of fluorescence emission of the bridged GFP chromophore is enhanced almost 60 -fold compared with that of the unbridged o-HBI. The inhibition of the excited-state deactivation channel via keto-type $S_{1} / S_{0}$ conical intersections makes the bridged GFP chromophore a potential fluorescence marker.

To further explore the time-dependent excited-state properties such as the $S_{1}$ lifetime, the deactivation processes, and the interplay of competitive excited-state channels, we carried out nonadiabatic surface-hopping dynamics simulations (275
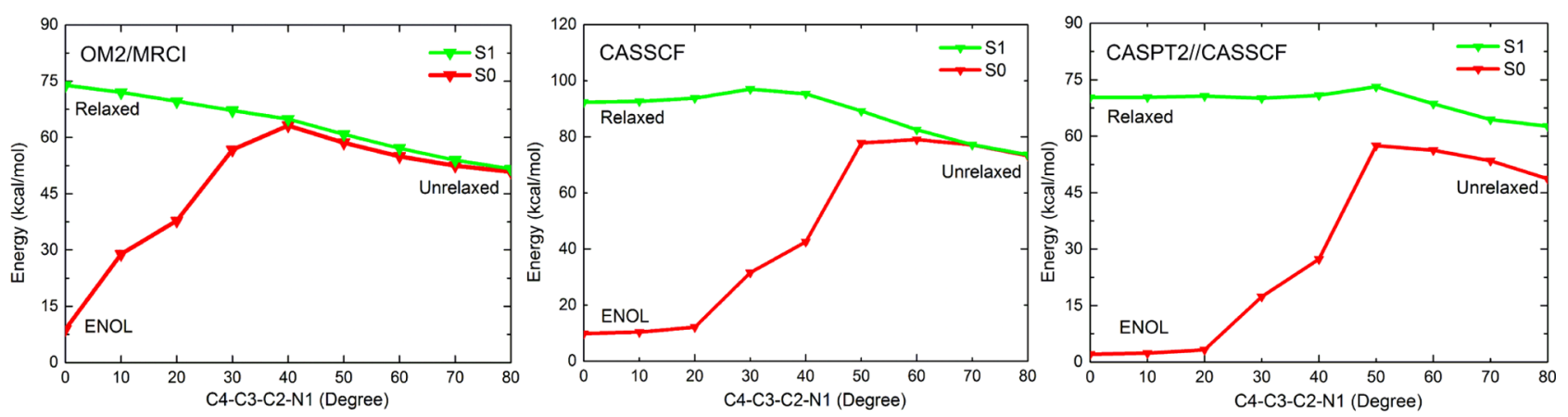

Figure 6. OM2/MRCI (left) and CASSCF (middle) computed minimum-energy deactivation paths connecting the $S_{1}$ enol minimum and the enol $S_{1} / S_{0}$ minimum-energy conical intersection (SOS1-ENOL-1) and corresponding MS-CASPT2//CASSCF energy profiles (right). See the text for discussion. 



Figure 7. OM2/MRCI (left) and CASSCF (middle) computed minimum-energy deactivation paths connecting the $S_{1}$ keto minimum and the keto $\mathrm{S}_{1} / \mathrm{S}_{0}$ minimum-energy conical intersection (SOS1-KETO-1) and corresponding MS-CASPT2//CASSCF energy profiles (right). See the text for discussion.

trajectories). They were performed using the semiempirical OM2/MRCI method, which was chosen because of its speed and its reliable performance in the static electronic structure calculations on the $S_{0}$ and $S_{1}$ potential energy surfaces of oLHBI (in comparison with CASSCF and MS-CASPT2, vide supra).

Excited-State Proton Transfer. In 217 of 261 successful trajectories, there is an excited-state intramolecular proton transfer (ESIPT) (see Table 3). This process is ultrafast and

Table 3. Final State of All Successful Trajectories at the End of 2 ps Simulation Time

\begin{tabular}{cccccc} 
& \multicolumn{3}{c}{$\mathrm{S}_{1}$} & & \multicolumn{2}{c}{$\mathrm{S}_{0}$} \\
\cline { 2 - 3 } \cline { 5 - 6 } total & enol & keto & & enol & keto \\
261 & 0 & 215 & & 44 & 2 \\
\hline
\end{tabular}

finished within 100 fs in nearly all trajectories (mostly within one vibrational period of ca. $50 \mathrm{fs}$; see the left panel of Figure 8). The top panel in Figure 9 (a, b) shows a typical trajectory. After excitation to the $S_{1}$ state in the Franck-Condon region, the $\mathrm{N} 1-\mathrm{H} 7$ distance quickly decreases and a covalent $\mathrm{N} 1-\mathrm{H} 7$ bond is formed within about 30 fs. During this ESIPT process, the system remains in the planar conformation: the two central C4C3C2N1 and $\mathrm{C} 5 \mathrm{C} 4 \mathrm{C} 3 \mathrm{C} 2$ dihedral angles vary only slightly, remaining typically within $20^{\circ}$ of the planar conformation (see panel $b$ in Figure 9). This kind of ultrafast ESIPT dynamics has been observed in many molecules with intramolecular hydrogen bonds. ${ }^{36,87-95}$ In the case of the typical trajectory that stays in the $S_{1}$ state for 2 ps (see top panel in Figure 9), the $S_{1}-S_{0}$ energy gap remains large during the entire simulation so that there is no hop (see Figure S7 in the Supporting Information).

The chemical locking of the rotation around the $\mathrm{C} 4-\mathrm{C} 3$ bond does not change the ESIPT dynamics. In our previous nonadiabatic dynamics simulations on the unlocked orthosubstituted GFP chromophore, ${ }^{41}$ the ESIPT process was also found to be ultrafast (tens of fs), and the central dihedral angles remained close to planarity. Thus, o-LHBI and o-HBI have similar ESIPT dynamics, which is essentially unaffected by the locking.

Excited-State Deactivation. However, the locking qualitatively changes the excited-state decay dynamics of o-LHBI compared with that of the unlocked o-HBI chromophore. First, in o-HBI, $90 \%$ of the trajectories are back in the $\mathrm{S}_{0}$ state at the end of the 2 ps simulation; ${ }^{41}$ by contrast, in o-LHBI, only $17 \%$ of the trajectories decay to the $S_{0}$ state within 2 ps, while $83 \%$ of the trajectories still stay around the keto $S_{1}$ minimum at the end of the simulation. This is qualitatively compatible with the experiments available: in toluene, the fluorescence quantum yield of the o-LHBDI derivative (dimethyl-substituted o-LHBI) is 0.18 , which is about 60 times higher than that of o-HBDI.

Second, in o-HBI, the trajectories return to the ground state mainly via the keto $S_{1} / S_{0}$ conical intersections in the cis region, while, in o-LHBI, this deactivation happens exclusively through the enol $S_{1} / S_{0}$ conical intersections. Figure 10 shows the distribution of selected distances and dihedral angles at all $S_{1} \rightarrow$ $\mathrm{S}_{0}$ hopping points in our dynamics simulations. As can be seen from the distribution of the $\mathrm{N} 1-\mathrm{H} 7$ and $\mathrm{O} 6-\mathrm{H} 7$ distances, almost all $\mathrm{S}_{1} \rightarrow \mathrm{S}_{0}$ hops occur in the enol region: the $\mathrm{H} 7$ atom is bonded to the $\mathrm{O} 6$ atom at all hopping points, and hence
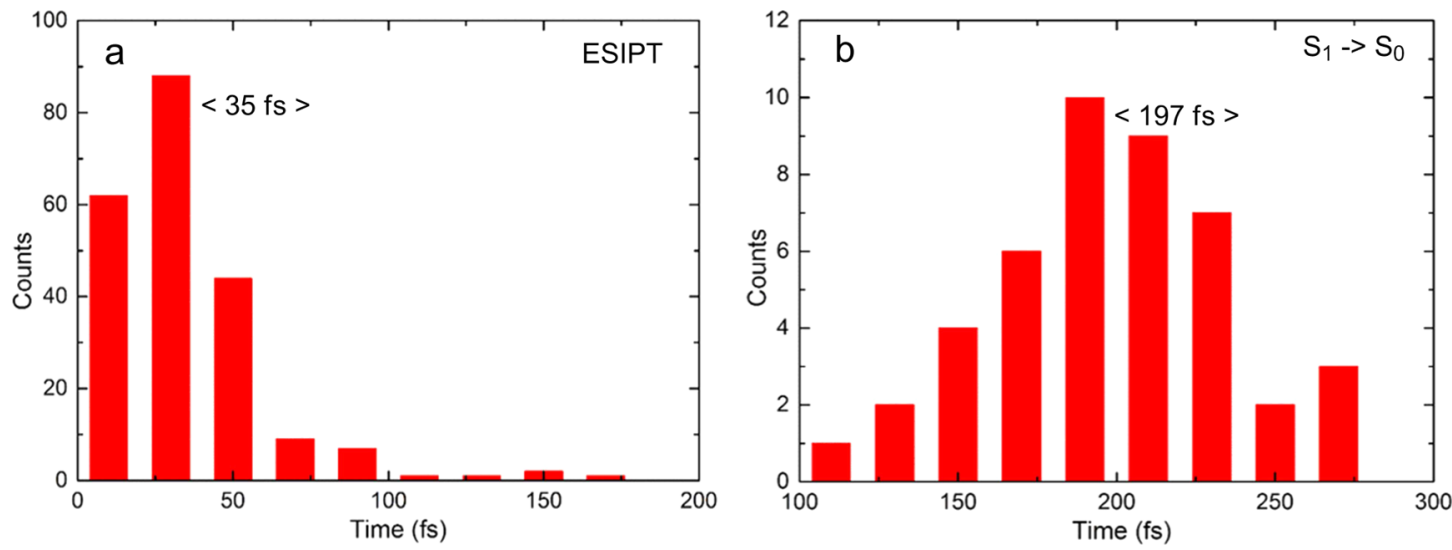

Figure 8. Time distributions of (a) the excited-state intramolecular proton transfer and (b) the $S_{1} \rightarrow S_{0}$ hopping events. See the text for discussion. 

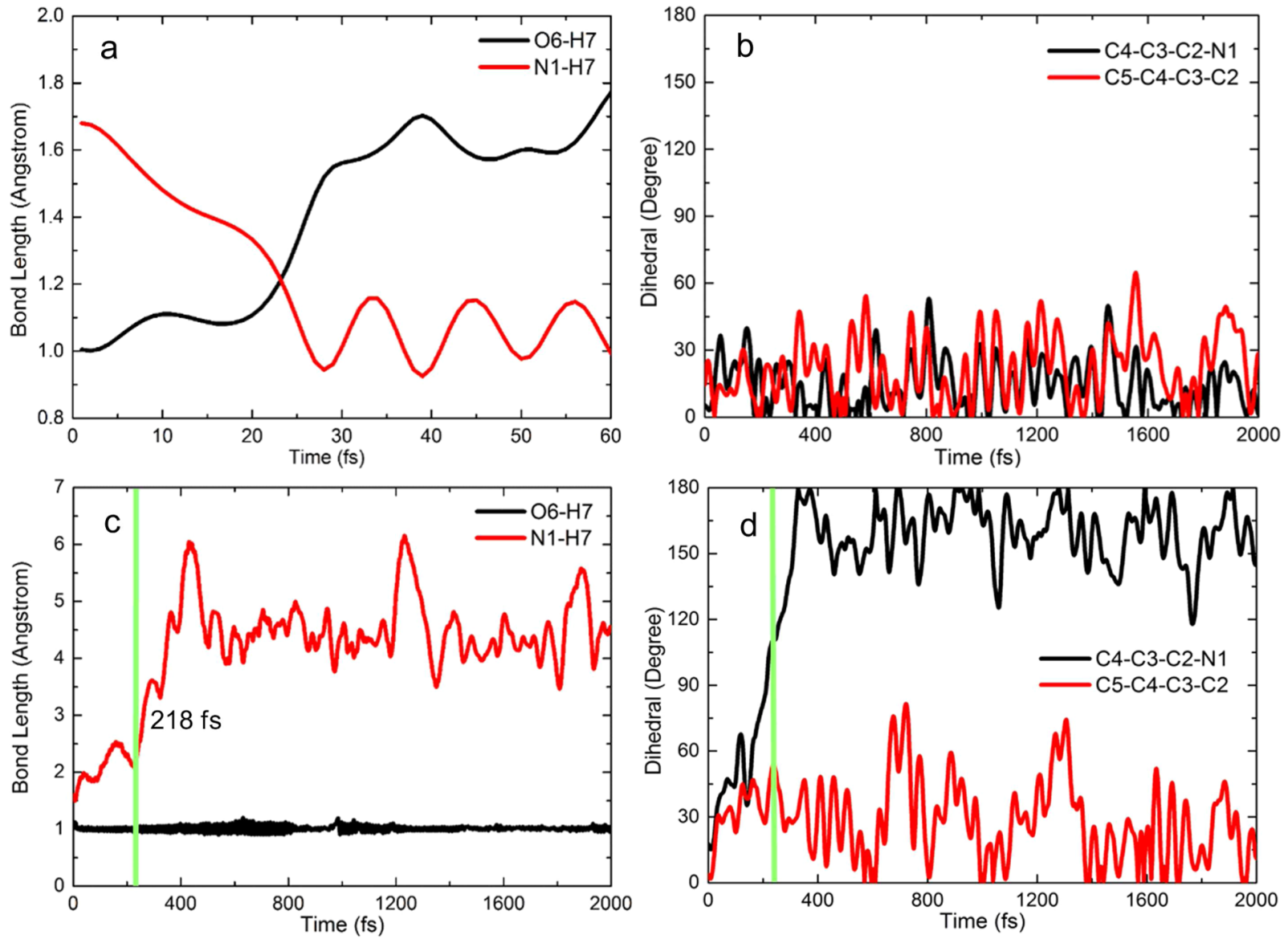

Figure 9. Time evolution of selected distances and dihedral angles during two typical trajectories: $(a, b)$ ultrafast excited-state proton transfer, the system remains in the $S_{1}$ state for 2 ps; (c, d) excited-state deactivation by hopping to the ground state after 218 fs. Note the different time scale on the top left. See the text for discussion.

there has been no prior excited-state intramolecular proton transfer. This is consistent with the computed excited-state topology. As discussed above, the enol $S_{1} / S_{0}$ conical intersection is low in energy (lying below the enol $S_{1}$ minimum, by $12.2 \mathrm{kcal} / \mathrm{mol}$ at the $\mathrm{MS}-\mathrm{CASPT} 2 / / \mathrm{CASSCF}$ level), and the deactivation path from the enol $S_{1}$ minimum to the enol $S_{1} / S_{0}$ conical intersection is barrierless (Figure 6). By contrast, the keto $S_{1} / S_{0}$ conical intersection lies $6.6 \mathrm{kcal} / \mathrm{mol}$ above the keto $S_{1}$ minimum (MS-CASPT2//CASSCF) and can only be reached via an uphill path (Figure 6). It is thus not surprising that the system decays preferentially through the enol $S_{1} / S_{0}$ conical intersection.

At the hopping points, the central $\mathrm{C} 4 \mathrm{C} 3 \mathrm{C} 2 \mathrm{~N} 1$ dihedral angle ranges between $40^{\circ}$ and $100^{\circ}$ (absolute values, Figure 10). This wide distribution reflects the topology of the $S_{1}$ and $S_{0}$ potential energy surfaces. As shown in Figure 6, the $S_{1}$ and $S_{0}$ energies are close to each other for $\mathrm{C} 4 \mathrm{C} 3 \mathrm{C} 2 \mathrm{~N} 1$ dihedral angles above $40^{\circ}$ (OM2/MRCI) or $50^{\circ}$ (CASSCF). Therefore, in the OM2/ MRCI nonadiabatic dynamics simulations, the $S_{1} \rightarrow S_{0}$ hopping region is quite extended, and hops occur at $\mathrm{C} 4 \mathrm{C} 3 \mathrm{C} 2 \mathrm{~N} 1$ dihedral angles far away from the optimized values of $98.7^{\circ}$ and $-98.1^{\circ}$ in the two enol-type minimum-energy conical intersections SOS1-ENOL-1 and SOS1-ENOL-2 (see Figure 5). This mismatch illustrates the need for full-dimensional simulations to arrive at a realistic photodynamics scenario.

The chemical locking in o-LHBI restrains the internal rotation around the $\mathrm{C} 3-\mathrm{C} 4$ bond during $\mathrm{S}_{1}$ excited-state deactivation. At the $S_{1} \rightarrow S_{0}$ hopping points, the associated
C5C4C3C2 dihedral angle is typically around $30^{\circ}$ (Figure 10) and is thus much smaller than the typical value of $60^{\circ}$ observed for the unlocked o-HBI GFP chromophore. ${ }^{41}$ Evidently, the rotation around the $\mathrm{C} 3-\mathrm{C} 4$ bond is restrained by the chemical locking but not completely suppressed, so that moderate C3C4 twists can still facilitate the excited-state deactivation to some extent.

The bottom panel of Figure 9 shows a typical trajectory for decay to the $S_{0}$ state via the enol $S_{1} / S_{0}$ conical intersection. Obviously this trajectory does not feature any excited-state intramolecular proton transfer since the $\mathrm{O} 6-\mathrm{H} 7$ bond length remains around its initial value of ca. $1.0 \AA$ during the whole simulation (black line). In the first $200 \mathrm{fs}$, the C4C3C2N1 dihedral angle quickly increases from small values to around $90^{\circ}$ thus moving the system toward the conical intersection region; the $S_{1} \rightarrow S_{0}$ hop occurs at $218 \mathrm{fs}$, and thereafter the $\mathrm{C} 4 \mathrm{C} 3 \mathrm{C} 2 \mathrm{~N} 1$ dihedral angle continues to rise toward $180^{\circ}$ in the ground state (cis-trans isomerization). The $\mathrm{C} 5 \mathrm{C} 4 \mathrm{C} 3 \mathrm{C} 2$ dihedral angle remains rather small during the entire simulation (with fluctuations reflecting the $\mathrm{C} 3-\mathrm{C} 4$ torsions).

Photochemical Mechanism. On the basis of our computational results, we propose the following photochemical mechanism for the o-LHBI chromophore (Figure 11). After photoexcitation into the first electronically excited $S_{1}$ state in the Franck-Condon region, the molecule quickly relaxes into a metastable $S_{1}$ enol minimum, which has two photochemical pathways available. (P-I) The first option is an excited-state intramolecular proton transfer leading to the $S_{1}$ keto species. It 

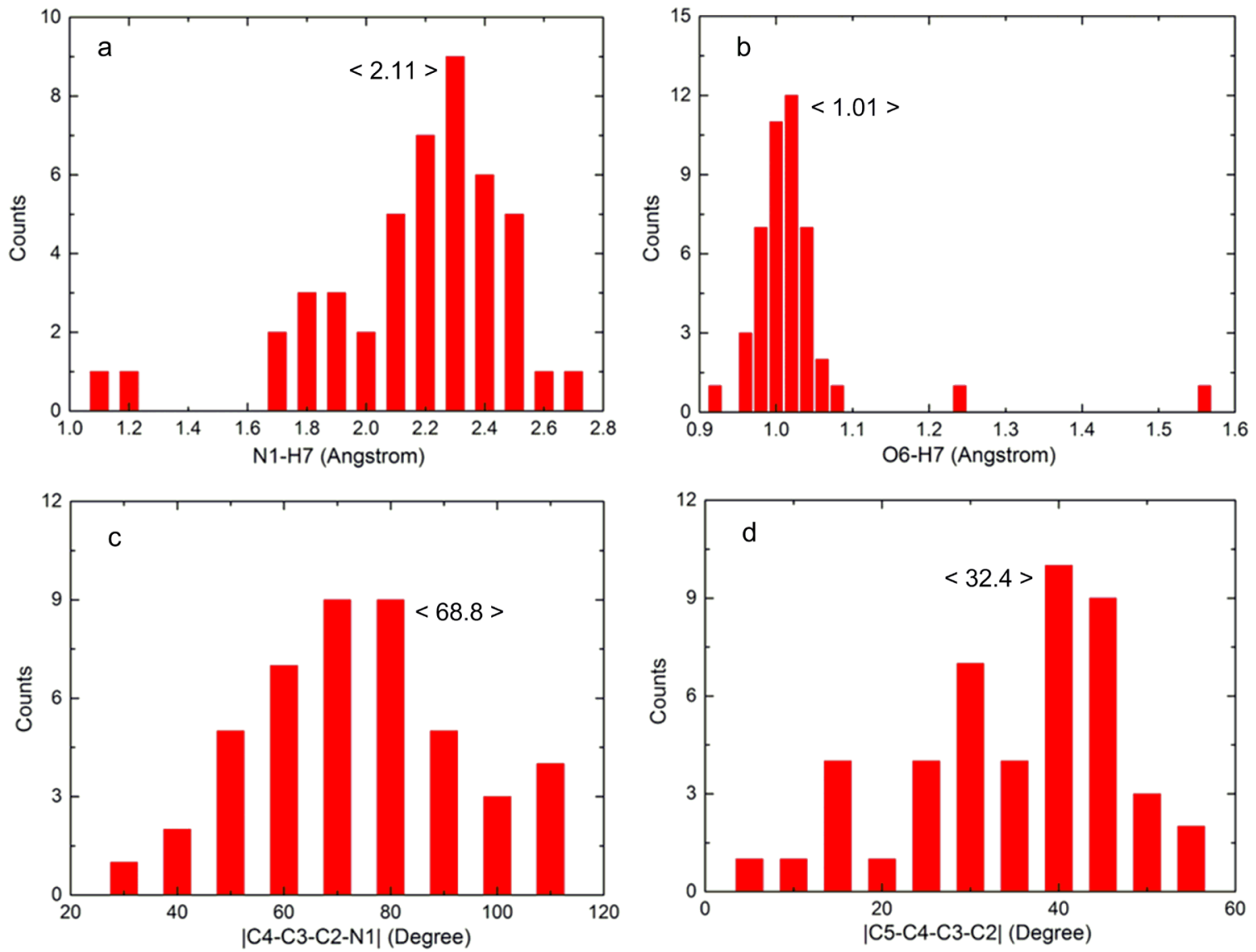

Figure 10. Distributions of the selected geometric parameters at the $S_{1} \rightarrow S_{0}$ hopping points. Average values are given in brackets. See the text for discussion.



Figure 11. Photochemical mechanism derived from the present static electronic structure calculations and nonadiabatic dynamics simulations. See the text for discussion.

is ultrafast (within $50 \mathrm{fs}$; OM2/MRCI). Upon arrival at the $S_{1}$ keto minimum, the trajectories are trapped because the forward deactivation channel to the keto $S_{1} / S_{0}$ conical intersection and the reverse excited-state proton transfer pathway back to the metastable $S_{1}$ enol minimum are both not accessible energetically (uphill processes). As a result, the $S_{1}$ keto species will be able to fluoresce with a large Stokes shift. (P-II) The second pathway is the direct $S_{1}$ excited-state deactivation via the enol $S_{1} / S_{0}$ conical intersection. This is also an efficient and exothermic process; its time scale is predicted to be about $200 \mathrm{fs}$ in the OM2/MRCI simulations.

P-I is the major pathway for the initial photochemistry of the o-LHBI chromophore because it is faster than the alternative $\mathbf{P}$ II. This is mainly because the excited-state intramolecular proton transfer takes place essentially within the molecular plane, whereas the competing isomerization via the enol $S_{1} / S_{0}$ conical intersection involves a large conformational change from the planar to the perpendicular conformation. Most importantly, the initial $S_{0}$ enol equilibrium structure has a planar structure with a strong $\mathrm{N} 1 \cdots \mathrm{H} 7-\mathrm{O} 6$ hydrogen bond; hence, its motion in the $S_{0}$ state will mainly sample geometries that remain close to planarity (with very small skeletal deformations). The initial conditions for the $S_{1}$ nonadiabatic dynamics are prepared from $S_{0} M D$ simulations, which tend to favor the part of phase space that is primarily spanned by the inplane coordinates (positions and momenta). As a consequence, the out-of-plane vibrational modes will be scarcely sampled, and there will thus be not much initial velocity in these modes, including the one for the $\mathrm{C} 4 \mathrm{C} 3 \mathrm{C} 2 \mathrm{~N} 1$ torsion. This situation is reflected in the results of our dynamics simulations. Only 44 out of 261 trajectories undergo an internal rotation around the $\mathrm{C} 2-\mathrm{C} 3$ bond to decay to the $\mathrm{S}_{0}$ state within the 2 ps simulations. In comparison, the excited-state proton transfer is observed in 215 trajectories, which are then trapped in the $S_{1}$ keto region until the end of the 2 ps simulations. Such trapping should significantly enhance the fluorescence quantum yield, in line with the experimental findings.

\section{CORRELATION WITH EXPERIMENTS}

Why does the chemical locking have only a slight effect on the fluorescence emission of p-LHBDI compared with unlocked pHBDI? For the GFP core chromophores with a para-hydroxyl group, the $S_{1}$ enol species is the only possible emitter. Hence, 

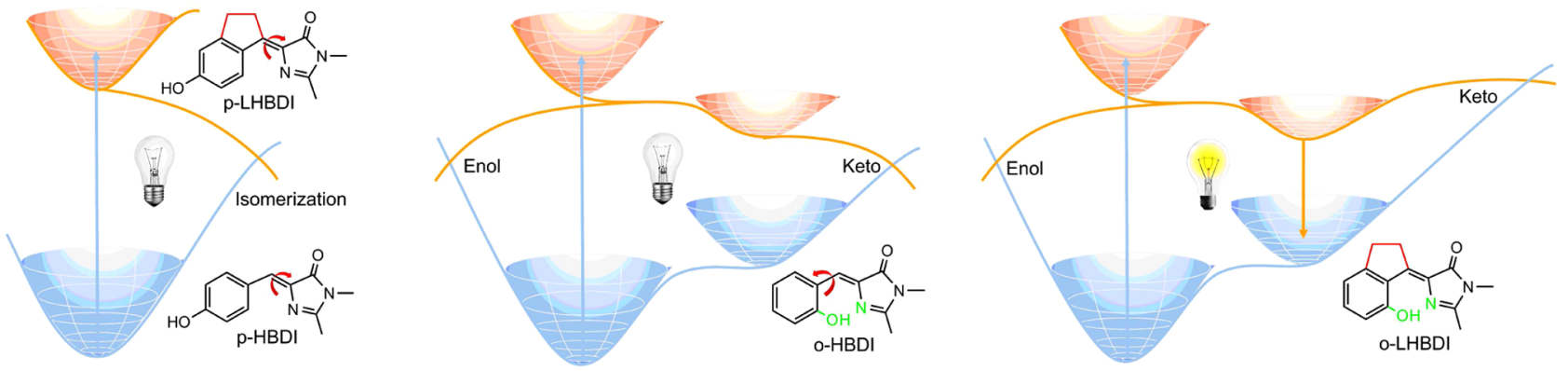

Figure 12. Comparison of photophysical and photochemical mechanisms of three types of GFP core chromophores. p-HBDI and p-LHBDI are nonemissive in vacuo and solution due to excited-state deactivation induced by cis-trans isomerization. o-HBDI is nonemissive because of ESIPTinduced excited-state deactivation. In o-LHBDI ESIPT leads to an excited-state trapping that enhances the ability of the $S_{1}$ keto species to fluoresce.

the quantum yield of fluorescence emission is interrelated with whether the $S_{1}$ enol decay channel can be easily accessed or not. The constraint on the $\mathrm{C} 3-\mathrm{C} 4$ torsion in $\mathrm{p}$-LHBDI has only a minor influence on the $S_{1}$ and $S_{0}$ potential energy profiles relevant to cis-trans isomerization. Therefore, both in p-HBDI and p-LHBDI, the excited-state deactivation induced by cis-trans isomerization is efficient and ultrafast in vacuo and solution (see the left panel of Figure 12). Hence, ultralow quantum yields of fluorescence emission are observed for both the p-LHBDI and p-HBDI chromophores.

Experimentally, the chemical locking of $\mathrm{C} 3-\mathrm{C} 4$ bond rotation enhances the fluorescence emission of o-LHBDI almost 60 -fold. This can be traced back to differences in the $S_{1}$ and $S_{0}$ potential energy surfaces of the locked o-LHBI and the unlocked o-HBI chromophores. The locking does not change the overall shape of these surfaces in the regions relevant to the enol-keto tautomerization, and hence the ESIPT processes are computed to be essentially barrierless and ultrafast in both oLHBI and o-HBI (on average within $35 \mathrm{fs}$ ). ${ }^{41}$ The subsequent excited-state deactivation of the $S_{1}$ keto species is also an essentially barrierless process (see the middle panel of Figure 12) and is thus ultrafast in o-HBI; as a consequence, the quantum yield of fluorescence emission of o-HBDI is about 3.3 $\times 10^{-3}$ experimentally (in toluene). ${ }^{39}$ In o-LHBI, by contrast, the locking blocks the excited-state deactivation channel from the $S_{1}$ keto minimum $S 1-K E T O$ to the keto $S_{1} / S_{0}$ conical intersections S0S1-KETO-1 and S0S1-KETO-2 (see the right panel of Figure 12); this increases the lifetime of the $S_{1}$ keto species and enhances its ability to fluoresce.

It is noteworthy that the cis-trans isomerization pathway cannot compete with the excited-state intramolecular proton transfer in the GFP core chromophores with an ortho-hydroxyl group, e.g. o-HBI and o-LHBI. In these compounds, the $S_{1}$ enol-keto tautomerization is complete within several tens of femtoseconds after irradiation; thus, the fluorescence emission mainly comes from these $S_{1}$ keto species. Therefore, blocking the $S_{1}$ keto decay channel is crucial for enhancing the fluorescence emission of these chromophores. In addition, if the locked o-LHBI chromophore is incorporated into GFPs, the cis-trans isomerization channel is expected to be suppressed further because of the steric constraints from the protein environment. These kinds of locked core chromophores should thus exhibit an even more enhanced fluorescence emission in GFPs.

The main purpose of our present work is to provide a qualitative explanation why the fluorescence quantum yield of the locked ortho-GFP chromophore is larger than that of its unlocked analogue $\left(0.18\right.$ vs $\left.3.3 \times 10^{-3}\right) .^{39,40}$ Our previous work has shown that there is a barrierless ESIPT-induced deactivation channel for the unlocked ortho-GFP chromophore. ${ }^{41}$ In the present static electronic structure calculations on the locked ortho-GFP chromophore we find that there is a barrier of about $8 \mathrm{kcal} / \mathrm{mol}$ on the path to the $S_{1} / S_{0}$ keto conical intersection, which prevents the efficient deactivation of the $S_{1}$ keto species (Figure 7). Therefore, it is clear qualitatively that the quantum yield of fluorescence emission of the locked chromophore is qualitatively larger than that of the unlocked one. Our dynamics simulations verify that the barrier indeed significantly delays the decay of the $S_{1}$ keto species, since most of the trajectories are trapped in this region.

Finally, we note that there are indeed a number of previous theoretical studies that address the competition between ESIPT and photoisomerization. ${ }^{89-91,93}$ However, to our knowledge, in-depth studies including nonadiabatic dynamics simulations have not yet been reported on this kind of competition in geometrically constrained chromophores like the one studied here. In our discussion, we have therefore focused on the orthoand para-substituted GFP chromophores with and without geometric constraints, in order to understand their different behavior, rather than attempting comprehensive comparisons with other systems.

\section{CONCLUSIONS}

In this computational study we explain the unusual excited-state behavior of chemically locked GFP core chromophores. Experimentally, chemical locking increases the quantum yield of fluorescence emission of GFP chromophores with an orthohydroxyl group, while it makes no difference to the quantum yield of fluorescence emission of GFP chromophores with a para-hydroxyl group. We use high-level electronic structure calculations to explore the $S_{1}$ and $S_{0}$ potential energy profiles of the intramolecular proton transfer, the excited-state deactivation channel, and the cis-trans isomerization pathway of a locked GFP model chromophore with an ortho-hydroxyl group (o-LHBI). We verify the proposed photophysical and photochemical mechanism derived from these static calculations by surface-hopping nonadiabatic dynamics simulations. On the basis of our previous and present results, we reveal the physical origin responsible for the enhancement of the quantum yield of fluorescence emission of o-LHBI, and we rationalize why chemical locking only changes the photophysics of the orthosubstituted GFP chromophores (and not of the parasubstituted ones). We hope that the present theoretical study will set the stage for knowledge-based improvement and design of GFP chromophores with better luminescence performance. 


\section{ASSOCIATED CONTENT}

\section{S Supporting Information}

The Supporting Information is available free of charge on the ACS Publications website at DOI: 10.1021/acs.jctc.5b00894.

Active orbitals in OM2/MRCI and CASSCF computations, additional figures and tables, and Cartesian coordinates of all optimized structures (PDF)

\section{AUTHOR INFORMATION}

\section{Corresponding Authors}

*E-mail: ganglong.cui@bnu.edu.cn.

*E-mail: thiel@mpi-muelheim.mpg.de.

\section{Notes}

The authors declare no competing financial interest.

\section{ACKNOWLEDGMENTS}

This work was supported by National Natural Science Foundation of China (Grants NSFC21522302 and NSFC21520102005); G.C. is also grateful for financial support from the "Recruitment Program of Global Youth Experts", the "Youth Scholars Program of Beijing Normal University", and the "Fundamental Research Funds for Central Universities"; W.T. acknowledges support from an ERC Advanced Grant (OMSQC).

\section{REFERENCES}

(1) Shimomura, O.; Johnson, F. H.; Saiga, Y. Extraction, Purification and Properties of Aequorin, a Bioluminescent Protein from the Luminous Hydromedusan, Aequorea. J. Cell. Comp. Physiol. 1962, 59, 223-239.

(2) Chalfie, M.; Tu, Y.; Euskirchen, G.; Ward, W. W.; Prasher, D. C. Green Fluorescent Protein as a Marker for Gene Expression. Science 1994, 263, 802-805.

(3) Cubitt, A. B.; Heim, R.; Adams, S. R.; Boyd, A. E.; Gross, L. A.; Tsien, R. Y. Understanding, Improving and Using Green Fluorescent Proteins. Trends Biochem. Sci. 1995, 20, 448-455.

(4) Tsien, R. Y. The Green Fluorescent Protein. Annu. Rev. Biochem. 1998, 67, 509-544.

(5) Shimomura, O. Discovery of Green Fluorescent Protein (GFP) (Nobel Lecture). Angew. Chem., Int. Ed. 2009, 48, 5590-5602.

(6) Chalfie, M. GFP: Lighting up Life (Nobel Lecture). Angew. Chem., Int. Ed. 2009, 48, 5603-5611.

(7) Tsien, R. Y. Constructing and Exploiting the Fluorescent Protein Paintbox (Nobel Lecture). Angew. Chem., Int. Ed. 2009, 48, 56125626.

(8) Zimmer, M. Green Fluorescent Protein (GFP): Applications, Structure, and Related Photophysical Behavior. Chem. Rev. 2002, 102, $759-782$.

(9) Nienhaus, G. U. The Green Fluorescent Protein: A Key Tool to Study Chemical Processes in Living Cells. Angew. Chem., Int. Ed. 2008, 47, 8992-8994.

(10) Schellenberg, P.; Johnson, E.; Esposito, A. P.; Reid, P. J.; Parson, W. W. Resonance Raman Scattering by the Green Fluorescent Protein and an Analogue of Its Chromophore. J. Phys. Chem. B 2001, 105, 5316-5322.

(11) Altoe', P.; Bernardi, F.; Garavelli, M.; Orlandi, G.; Negri, F. Solvent Effects on the Vibrational Activity and Photodynamics of the Green Fluorescent Protein Chromophore: A Quantum-Chemical Study. J. Am. Chem. Soc. 2005, 127, 3952-3963.

(12) Martin, M. E.; Negri, F.; Olivucci, M. Origin, Nature, and Fate of the Fluorescent State of the Green Fluorescent Protein Chromophore at the CASPT2//CASSCF Resolution. J. Am. Chem. Soc. 2004, 126, 5452-5464.

(13) Sinicropi, A.; Andruniow, T.; Ferré, N.; Basosi, R.; Olivucci, M. Properties of the Emitting State of the Green Fluorescent Protein
Resolved at the CASPT2//CASSCF/CHARMM Level. J. Am. Chem. Soc. 2005, 127, 11534-11535.

(14) Dong, J.; Solntsev, K. M.; Tolbert, L. M. Solvatochromism of the Green Fluorescence Protein Chromophore and Its Derivatives. J. Am. Chem. Soc. 2006, 128, 12038-12039.

(15) Vendrell, O.; Gelabert, R.; Moreno, M.; Lluch, J. M. Potential Energy Landscape of the Photoinduced Multiple Proton-Transfer Process in the Green Fluorescent Protein: Classical Molecular Dynamics and Multiconfigurational Electronic Structure Calculations. J. Am. Chem. Soc. 2006, 128, 3564-3574.

(16) Dong, J.; Solntsev, K. M.; Poizat, O.; Tolbert, L. M. The MetaGreen Fluorescent Protein Chromophore. J. Am. Chem. Soc. 2007, 129, 10084-10085.

(17) Polyakov, I. V.; Grigorenko, B. L.; Epifanovsky, E. M.; Krylov, A. I.; Nemukhin, A. V. Potential Energy Landscape of the Electronic States of the GFP Chromophore in Different Protonation Forms: Electronic Transition Energies and Conical Intersections. J. Chem. Theory Comput. 2010, 6, 2377-2387.

(18) Bravaya, K. B.; Khrenova, M. G.; Grigorenko, B. L.; Nemukhin, A. V.; Krylov, A. I. Effect of Protein Environment on Electronically Excited and Ionized States of the Green Fluorescent Protein Chromophore. J. Phys. Chem. B 2011, 115, 8296-8303.

(19) Addison, K.; Conyard, J.; Dixon, T.; Bulman Page, P. C.; Solntsev, K. M.; Meech, S. R. Ultrafast Studies of the Photophysics of Cis and Trans States of the Green Fluorescent Protein Chromophore. J. Phys. Chem. Lett. 2012, 3, 2298-2302.

(20) Bravaya, K. B.; Krylov, A. I. On the Photodetachment from the Green Fluorescent Protein Chromophore. J. Phys. Chem. A 2013, 117, 11815-11822.

(21) West, C. W.; Hudson, A. S.; Cobb, S. L.; Verlet, J. R. Communication: Autodetachment Versus Internal Conversion from the $S_{1}$ State of the Isolated GFP Chromophore Anion. J. Chem. Phys. 2013, 139, 071104.

(22) Ding, L.; Chung, L. W.; Morokuma, K. Reaction Mechanism of Photoinduced Decarboxylation of the Photoactivatable Green Fluorescent Protein: An ONIOM (QM: MM) Study. J. Phys. Chem. B 2013, 117, 1075-1084.

(23) Grigorenko, B. L.; Nemukhin, A. V.; Polyakov, I. V.; Morozov, D. I.; Krylov, A. I. First-Principles Characterization of the Energy Landscape and Optical Spectra of Green Fluorescent Protein Along the $\mathrm{A} \rightarrow \mathrm{I} \rightarrow \mathrm{B}$ Proton Transfer Route. J. Am. Chem. Soc. 2013, 135, $11541-11549$.

(24) Deng, S. H. M.; Kong, X.-Y.; Zhang, G.; Yang, Y.; Zheng, W.-J.; Sun, Z.-R.; Zhang, D.-Q.; Wang, X.-B. Vibrationally Resolved Photoelectron Spectroscopy of the Model GFP Chromophore Anion Revealing the Photoexcited $S_{1}$ State Being Both Vertically and Adiabatically Bound against the Photodetached $\mathrm{D}_{0}$ Continuum. J. Phys. Chem. Lett. 2014, 5, 2155-2159.

(25) Thompson, L. M.; Lasoroski, A.; Champion, P. M.; Sage, J. T.; Frisch, M. J.; van Thor, J. J.; Bearpark, M. J. Analytical Harmonic Vibrational Frequencies for the Green Fluorescent Protein Computed with ONIOM: Chromophore Mode Character and Its Response to Environment. J. Chem. Theory Comput. 2014, 10, 751-766.

(26) Zhang, Q.; Chen, X.; Cui, G. L.; Fang, W.-H.; Thiel, W. Concerted Asynchronous Hula-Twist Photoisomerization in the S65T/H148D Mutant of Green Fluorescent Protein. Angew. Chem., Int. Ed. 2014, 53, 8649-8653.

(27) Armengol, P.; Gelabert, R.; Moreno, M.; Lluch, J. M. Unveiling How an Archetypal Fluorescent Protein Operates: Theoretical Perspective on the Ultrafast Excited State Dynamics of GFP Variant S65T/H148D. J. Phys. Chem. B 2015, 119, 2274-2291.

(28) Send, R.; Suomivuori, C.-M.; Kaila, V. R. I.; Sundholm, D. Coupled-Cluster Studies of Extensive Green Fluorescent Protein Models Using the Reduced Virtual Space Approach. J. Phys. Chem. B 2015, 119, 2933-2945.

(29) Grigorenko, B. L.; Nemukhin, A. V.; Polyakov, I. V.; Khrenova, M.; Krylov, A. I. A Light-Induced Reaction with Oxygen Leads to Chromophore Decomposition and Irreversible Photobleaching in GFP-Type Proteins. J. Phys. Chem. B 2015, 119, 5444-5452. 
(30) Toniolo, A.; Granucci, G.; Martínez, T. J. Conical Intersections in Solution: A QM/MM Study using Floating Occupation Semiempirical Configuration Interaction Wave Functions. J. Phys. Chem. A 2003, 107, 3822-3830.

(31) Stavrov, S. S.; Solntsev, K. M.; Tolbert, L. M.; Huppert, D. Probing the Decay Coordinate of the Green Fluorescent Protein: Arrest of Cis-Trans Isomerization by the Protein Significantly Narrows the Fluorescence Spectra. J. Am. Chem. Soc. 2006, 128, 1540-1546.

(32) Schäfer, L. V.; Groenhof, G.; Klingen, A. R.; Ullmann, G. M.; Boggio-Pasqua, M.; Robb, M. A.; Grubmüller, H. Photoswitching of the Fluorescent Protein asFP595 Mechanism, Proton Pathways, and Absorption Spectra. Angew. Chem. 2007, 119, 536-542.

(33) Olsen, S.; Smith, S. C. Radiationless Decay of Red Fluorescent Protein Chromophore Models via Twisted Intramolecular ChargeTransfer States. J. Am. Chem. Soc. 2007, 129, 2054-2065.

(34) Olsen, S.; Smith, S. C. Bond Selection in the Photoisomerization Reaction of Anionic Green Fluorescent Protein and Kindling Fluorescent Protein Chromophore Models. J. Am. Chem. Soc. 2008, 130, 8677-8689.

(35) Forbes, M. W.; Jockusch, R. A. Deactivation Pathways of an Isolated Green Fluorescent Protein Model Chromophore Studied by Electronic Action Spectroscopy. J. Am. Chem. Soc. 2009, 131, 1703817039.

(36) Olsen, S.; Lamothe, K.; Martínez, T. J. Protonic Gating of Excited-State Twisting and Charge Localization in GFP Chromophores: A Mechanistic Hypothesis for Reversible Photoswitching. J. Am. Chem. Soc. 2010, 132, 1192-1193.

(37) Chen, K.-Y.; Cheng, Y.-M.; Lai, C.-H.; Hsu, C.-C.; Ho, M.-L.; Lee, G.-H.; Chou, P.-T. Ortho Green Fluorescence Protein Synthetic Chromophore; Excited-State Intramolecular Proton Transfer via a Seven-Membered-Ring Hydrogen-Bonding System. J. Am. Chem. Soc. 2007, 129, 4534-4535.

(38) Kang, J.; Zhao, G.; Xu, J.; Yang, W. A Synthesized GFP Analogue Emits via a Hydrogen-Bonding System. Chem. Commun. 2010, 46, 2868-2870.

(39) Hsieh, C.-C.; Chou, P.-T.; Shih, C.-W.; Chuang, W.-T.; Chung, M.-W.; Lee, J.; Joo, T. Comprehensive Studies on an Overall Proton Transfer Cycle of the ortho-Green Fluorescent Protein Chromophore. J. Am. Chem. Soc. 2011, 133, 2932-2943.

(40) Hsu, Y.-H.; Chen, Y.-A.; Tseng, H.-W.; Zhang, Z.; Shen, J.-Y.; Chuang, W.-T.; Lin, T.-C.; Lee, C.-S.; Hung, W.-Y.; Hong, B.-C.; Liu, S.-H.; Chou, P.-T. Locked ortho- and para-Core Chromophores of Green Fluorescent Protein; Dramatic Emission Enhancement via Structural Constraint. J. Am. Chem. Soc. 2014, 136, 11805-11812.

(41) Cui, G. L.; Lan, Z.; Thiel, W. Intramolecular Hydrogen Bonding Plays a Crucial Role in the Photophysics and Photochemistry of the GFP Chromophore. J. Am. Chem. Soc. 2012, 134, 1662-1672.

(42) Parr, R. G.; Yang, W. T. Density-Functional Theory of Atoms and Molecules; Oxford University Press: USA, 1994.

(43) Vosko, S. H.; Wilk, L.; Nusair, M. Accurate Spin-Dependent Electron Liquid Correlation Energies for Local Spin Density Calculations: A Critical Analysis. Can. J. Phys. 1980, 58, 1200-1211.

(44) Lee, C.; Yang, W. T.; Parr, R. G. Development of the ColleSalvetti Correlation-Energy Formula into a Functional of the Electron Density. Phys. Rev. B: Condens. Matter Mater. Phys. 1988, 37, 785-789.

(45) Becke, A. D. Density-Functional Exchange-Energy Approximation with Correct Asymptotic Behavior. Phys. Rev. A: At., Mol., Opt. Phys. 1988, 38, 3098-3100.

(46) Becke, A. D. A New Mixing of Hartree-Fock and Local DensityFunctional Theories. J. Chem. Phys. 1993, 98, 1372-1377.

(47) Cui, G. L.; Guan, P.-J.; Fang, W.-H. Photoinduced Proton Transfer and Isomerization in a Hydrogen-Bonded Aromatic Azo Compound: A CASPT2//CASSCF Study. J. Phys. Chem. A 2014, 118, 4732-4739.

(48) Guan, P.-J.; Cui, G.; Fang, Q. Computational Photochemistry of the Azobenzene Scaffold of Sudan I and Orange II Dyes: Excited-State Proton Transfer and Deactivation via Conical Intersections. ChemPhysChem 2015, 16, 805-811.
(49) Andersson, K.; Malmqvist, P.-Å; Roos, B. O.; Sadlej, A. J.; Wolinski, K. Second-Order Perturbation Theory with a CASSCF Reference Function. J. Phys. Chem. 1990, 94, 5483-5488.

(50) Andersson, K.; Malmqvist, P.-Å; Roos, B. O. Second-Order Perturbation Theory with a Complete Active Space Self-Consistent Field Reference Function. J. Chem. Phys. 1992, 96, 1218-1226.

(51) Aquilante, F.; Lindh, R.; Pedersen, T. B. Unbiased Auxiliary Basis Sets for Accurate Two-Electron Integral Approximations. J. Chem. Phys. 2007, 127, 114107.

(52) Ghigo, G.; Roos, B. O.; Malmqvist, P.-Å. A Modified Definition of the Zeroth-Order Hamiltonian in Multiconfigurational Perturbation Theory (CASPT2). Chem. Phys. Lett. 2004, 396, 142-149.

(53) Försberg, N.; Malmqvist, P.-Å. Multiconfiguration Perturbation Theory with Imaginary Level Shift. Chem. Phys. Lett. 1997, 274, 196204.

(54) Marques, M. A. L.; Ullrich, C. A.; Nogueira, F.; Rubio, A.; Burke, K.; Gross, E. K. U. Time-dependent Density Functional Theory; Springer: 2006

(55) Yanai, T.; Tew, D. P.; Handy, N. C. A New Hybrid ExchangeCorrelation Functional Using the Coulomb-Attenuated Method (CAM-B3LYP). Chem. Phys. Lett. 2004, 393, 51-57.

(56) Ditchfield, R.; Hehre, W. J.; Pople, J. A. Self-Consistent Molecular-Orbital Methods. IX. An Extended Gaussian-Type Basis for Molecular-Orbital Studies of Organic Molecules. J. Chem. Phys. 1971, $54,724-728$.

(57) Francl, M. M.; Pietro, W. J.; Hehre, W. J.; Binkley, J. S.; Gordon, M. S.; DeFrees, D. J.; Pople, J. A. Self-Consistent Molecular Orbital Methods. XXIII. A Polarization-Type Basis Set for Second-Row Elements. J. Chem. Phys. 1982, 77, 3654-3665.

(58) Frisch, M. J.; Trucks, G. W.; Schlegel, H. B.; Scuseria, G. E.; Robb, M. A.; Cheesem, J. R.; Scalmani, G.; Barone, V.; Mennucci, B.; Petersson, G. A.; Nakatsuji, H.; Caricato, M.; Li, X.; Hratchian, H. P.; Izmaylov, A. F.; Bloino, J.; Zheng, G.; Sonnenberg, J. L.; Hada, M.; Ehara, M.; Toyota, K.; Fukuda, R.; Hasegawa, J.; Ishida, M.; Nakajima, T.; Honda, Y.; Kitao, O.; Nakai, H.; Vreven, T.; Montgomery, J. A., Jr.; Peralta, J. E.; Ogliaro, F.; Bearpark, M.; Heyd, J. J.; Brothers, E.; Kudin, K. N.; Staroverov, V. N.; Kobayashi, R.; Normand, J.; Raghavachari, K.; Rendell, A.; Burant, J. C.; Iyengar, S. S.; Tomasi, J.; Cossi, M.; Rega, N.; Millam, J. M.; Klene, M.; Knox, J. E.; Cross, J. B.; Bakken, V.; Adamo, C.; Jaramillo, J.; Gomperts, R.; Stratmann, R. E.; Yazyev, O.; Austin, A. J.; Cammi, R.; Pomelli, C.; Ochterski, J. W.; Martin, R. L.; Morokuma, K.; Zakrzewski, V. G.; Voth, G. A.; Salvador, P.; Dannenberg, J. J.; Dapprich, S.; Daniels, A. D.; Farkas, O.; Foresman, J. B.; Ortiz, J. V.; Cioslowski, J.; Fox, D. J. Gaussian 09, Revision A.02; Gaussian, Inc.: Wallingford, CT, 2009.

(59) Karlström, G.; Lindh, R.; Malmqvist, P.-Å.; Roos, B. O.; Ryde, U.; Veryazov, V.; Widmark, P. O.; Cossi, M.; Schimmelpfennig, B.; Neogrady, P.; Seijo, L. MOLCAS: A Program Package for Computational Chemistry. Comput. Mater. Sci. 2003, 28, 222-229.

(60) Aquilante, F.; De Vico, L.; Ferré, N.; Ghigo, G.; Malmqvist, P.Å.; Neogrády, P.; Pedersen, T. B.; Pitoňák, M.; Reiher, M.; Roos, B. O.; Serrano-Andrés, L.; Urban, M.; Veryazov, V.; Lindh, R. MOLCAS 7: The Next Generation. J. Comput. Chem. 2010, 31, 224-247.

(61) Werner, H.-J.; Knowles, P. J.; Knizia, G.; Manby, F. R.; Schütz, M.; Celani, P.; Korona, T.; Lindh, R.; Mitrushenkov, A.; Rauhut, G.; Shamasundar, K. R.; Adler, T. B.; Amos, R. D.; Bernhardsson, A.; Berning, A.; Cooper, D. L.; Deegan, M. J. O.; Dobbyn, A. J.; Eckert, F.; Goll, E.; Hampel, C.; Hesselmann, A.; Hetzer, G.; Hrenar, T.; Jansen, G.; Köppl, C.; Liu, Y.; Lloyd, A. W.; Mata, R. A.; May, A. J.; McNicholas, S. J.; Meyer, W.; Mura, M. E.; Nicklass, A.; O’Neill, D. P.; Palmieri, P.; Peng, D.; Pflüger, K.; Pitzer, R; Reiher, M.; Shiozaki, T.; Stoll, H.; Stone, A. J.; Tarroni, R.; Thorsteinsson, T.; Wang, M. MOLPRO, version 2010.1, a package of ab initio programs. 2010. See: http://www.molpro.net (accessed December 11, 2015).

(62) Weber, W. Ph.D. Thesis, University of Zürich, 1996.

(63) Weber, W.; Thiel, W. Orthogonalization Corrections for Semiempirical Methods. Theor. Chem. Acc. 2000, 103, 495-506.

(64) Koslowski, A.; Beck, M. E.; Thiel, W. Implementation of a General Multireference Configuration Interaction Procedure with 
Analytic Gradients in a Semiempirical Context Using the Graphical Unitary Group Approach. J. Comput. Chem. 2003, 24, 714-726.

(65) Thiel, W. MNDO99 program, version 6.1; Max-Planck-Institut für Kohlenforschung: Mülheim, Germany, 2007.

(66) Yarkony, D. R. Nuclear Dynamics near Conical Intersections in the Adiabatic Representation: I. The Effects of Local Topography on Interstate Transitions. J. Chem. Phys. 2001, 114, 2601-2613.

(67) Keal, T. W.; Koslowski, A.; Thiel, W. Comparison of Algorithms for Conical Intersection Optimisation Using Semiempirical Methods. Theor. Chem. Acc. 2007, 118, 837-844.

(68) Tully, J. C.; Preston, R. K. Trajectory Surface Hopping Approach to Nonadiabatic Molecular Collisions: Reaction of $\mathrm{H}$ with D2. J. Chem. Phys. 1971, 55, 562-572.

(69) Hammes-Schiffer, S.; Tully, J. C. Proton Transfer in Solution: Molecular Dynamics with Quantum Transitions. J. Chem. Phys. 1994, 101, 4657-4667.

(70) Fabiano, E.; Keal, T. W.; Thiel, W. Implementation of Surface Hopping Molecular Dynamics Using Semiempirical Methods. Chem. Phys. 2008, 349, 334-347.

(71) Virshup, A. M.; Punwong, C.; Pogorelov, T. V.; Lindquist, B. A.; Ko, C.; Martínez, T. J. Photodynamics in Complex Environments: Ab Initio Multiple Spawning Quantum Mechanical/Molecular Mechanical Dynamics. J. Phys. Chem. B 2009, 113, 3280-3291.

(72) Tao, H.; Levine, B. G.; Martínez, T. J. Ab Initio Multiple Spawning Dynamics Using Multi-State Second-Order Perturbation Theory. J. Phys. Chem. A 2009, 113, 13656-13662.

(73) Barbatti, M.; Aquino, A. J. A.; Szymczak, J. J.; Nachtigallová, D.; Hobza, P.; Lischka, H. Relaxation mechanisms of UV-photoexcited DNA and RNA nucleobases. Proc. Natl. Acad. Sci. U. S. A. 2010, 107, 21453-21458.

(74) Nachtigallová, D.; Zelený, T.; Ruckenbauer, M.; Müller, T.; Barbatti, M.; Hobza, P.; Lischka, H. Does Stacking Restrain the Photodynamics of Individual Nucleobases? J. Am. Chem. Soc. 2010, 132, 8261-8263.

(75) Cui, G. L.; Ai, Y.; Fang, W. Conical Intersection Is Responsible for the Fluorescence Disappearance Below $365 \mathrm{~nm}$ in Cyclopropanone. J. Phys. Chem. A 2010, 114, 730-734.

(76) Cui, G. L.; Fang, W. Ab Initio Based Surface-Hopping Dynamics Study on Ultrafast Internal Conversion in Cyclopropanone. J. Phys. Chem. A 2011, 115, 1547-1555.

(77) Cui, G. L.; Fang, W. Ab Initio Trajectory Surface-Hopping Study on Ultrafast Deactivation Process of Thiophene. J. Phys. Chem. A 2011, 115, 11544-11550.

(78) Richter, M.; Marquetand, P.; González-Vázquez, J.; Sola, I.; González, L. Femtosecond Intersystem Crossing in the DNA Nucleobase Cytosine. J. Phys. Chem. Lett. 2012, 3, 3090-3095.

(79) Martínez-Fernández, L.; Corral, I.; Granucci, G.; Persico, M. Competing Ultrafast Intersystem Crossing and Internal Conversion: A Time Resolved Picture for the Deactivation of 6-Thioguanine. Chem. Sci. 2014, 5, 1336-1347.

(80) Cui, G. L.; Thiel, W. Generalized Trajectory Surface-Hopping Method for Internal Conversion and Intersystem Crossing. J. Chem. Phys. 2014, 141, 124101.

(81) Granucci, G.; Persico, M.; Zoccante, A. Including Quantum Decoherence in Surface Hopping. J. Chem. Phys. 2010, 133, 134111.

(82) Kazaryan, A.; Lan, Z. G.; Schäfer, L. V.; Thiel, W.; Filatov, M. Surface Hopping Excited-State Dynamics Study of the Photoisomerization of a Light-Driven Fluorene Molecular Rotary Motor. J. Chem. Theory Comput. 2011, 7, 2189-2199.

(83) Weingart, O.; Lan, Z. G.; Koslowski, A.; Thiel, W. Chiral Pathways and Periodic Decay in cis-Azobenzene Photodynamics. J. Phys. Chem. Lett. 2011, 2, 1506-1509.

(84) Spörkel, L.; Cui, G. L.; Koslowski, A.; Thiel, W. Nonequilibrium $\mathrm{H} /$ D Isotope Effects from Trajectory-Based Nonadiabatic Dynamics. J. Phys. Chem. A 2014, 118, 152-157.

(85) Xia, S.-H.; Xie, B.-B.; Fang, Q.; Cui, G. L.; Thiel, W. ExcitedState Intramolecular Proton Transfer to Carbon Atoms: Nonadiabatic Surface-Hopping Dynamics Simulations. Phys. Chem. Chem. Phys. 2015, 17, 9687-9697.
(86) Xie, B.-B.; Xia, S.-H.; Liu, L.-H.; Cui, G. L. Surface-Hopping Dynamics Simulations of Malachite Green: A Triphenylmethane Dye. J. Phys. Chem. A 2015, 119, 5607-5617.

(87) Sobolewski, A. L.; Domcke, W.; Hättig, C. Tautomeric selectivity of the excited-state lifetime of guanine/cytosine base pairs: The role of electron-driven proton-transfer processes. Proc. Natl. Acad. Sci. U. S. A. 2005, 102, 17903-17906.

(88) Schultz, T.; Samoylova, E.; Radloff, W.; Hertel, I. V.; Sobolewski, A. L.; Domcke, W. Efficient deactivation of a model base pair via excited-state hydrogen transfer. Science 2004, 306, 17651768.

(89) Coe, J. D.; Martínez, T. J. Competitive decay at two-and threestate conical intersections in excited-state intramolecular proton transfer. J. Am. Chem. Soc. 2005, 127, 4560-4561.

(90) Coe, J. D.; Martínez, T. J. Ab initio molecular dynamics of excited-state intramolecular proton transfer around a three-state conical intersection in malonaldehyde. J. Phys. Chem. A 2006, 110, 618-630.

(91) Barbatti, M.; Aquino, A. J. A.; Lischka, H.; Schriever, C.; Lochbrunner, S.; Riedle, E. Ultrafast internal conversion pathway and mechanism in 2-(2'-hydroxyphenyl) benzothiazole: a case study for excited-state intramolecular proton transfer systems. Phys. Chem. Chem. Phys. 2009, 11, 1406-1415.

(92) Daengngern, R.; Kungwan, N.; Wolschann, P.; Aquino, A. J. A.; Lischka, H.; Barbatti, M. Excited-state intermolecular proton transfer reactions of 7-azaindole $(\mathrm{MeOH})_{n}(\mathrm{n}=1-3)$ clusters in the gas phase: on-the-fly dynamics simulation. J. Phys. Chem. A 2011, 115, 1412914136.

(93) Karsili, T. N. V.; Marchetti, B.; Ashfold, M. N. R.; Domcke, W. $\mathrm{Ab}$ initio study of potential ultrafast internal conversion routes in oxybenzone, caffeic acid, and ferulic acid: Implications for sunscreens. J. Phys. Chem. A 2014, 118, 11999-12010.

(94) Kochman, M. A.; Morrison, C. A. Hybrid QM/QM simulations of excited-state intramolecular proton transfer in the molecular crystal 7-(2-pyridyl)-indole. J. Chem. Theory Comput. 2013, 9, 1182-1192.

(95) Ding, L. N.; Chung, L. W.; Morokuma, K. J. Excited-State Proton Transfer Controls Irreversibility of Photoisomerization in Mononuclear Ruthenium (II) Monoaquo Complexes: A DFT Study. J. Chem. Theory Comput. 2014, 10, 668-675. 